DOI 10.31489/2019No2/105-112

UDC $517.958+004.942+533.9$

\title{
ON THE KERNEL PROPERTIES OF THE INTEGRAL EQUATION FOR THE MODEL WITH THE ESSENTIALLY LOADED HEAT EQUATION
}

\author{
Yesbayev A.N. ${ }^{1}$, Yessenbayeva G.A. ${ }^{2}$, Yessenbayeva G.A. ${ }^{3}$, Ramazanov M.I. ${ }^{2}$ \\ 1'L.N. Gumilyov Eurasian National University, Nur-Sultan, Kazakhstan \\ 2E.A. Buketov Karaganda State University, Karaganda, Kazakhstan, esenbaevagulsima@mail.ru \\ ${ }^{3}$ Karaganda Economic University of Kazpotrebsoyuz, Karaganda, Kazakhstan
}

\begin{abstract}
Mathematical modeling of thermophysical processes in an electric arc of high-current disconnecting apparatuses leads to a boundary value problem for an essentially loaded heat conduction equation. Taking into account the transience of such phenomena, in some cases only a mathematical model is able to give adequate information about their dynamics. The mathematical model in the form of the boundary value problem is reduced to the Volterra integral equation of the second kind, as a result, we have that the solvability of the boundary value problem is equivalent to the solvability of the reduced integral equation. Thus, there is a need to study the reduced integral equation. The results of this study (various representations and properties of the kernel-forming function in general case and the types of the kernel of the integral equation in special cases) are presented in this article. The article is focused at physicists and engineers, as well as scientific researchers engaged in the practical applications of loaded differential equations.
\end{abstract}

Keywords: thermophysical processes, electric arc, loaded heat equation, boundary value problem, reduced integral equation, kernel of an integral equation.

\section{Introduction}

The modern trend in technology to use super-strong and super-weak currents in many electrical devices leads to the need to study phenomena outside the usual current range. When switching electrical devices or overvoltage an electric arc may appear in the circuit between the currentcarrying parts [1]. To ignite the arc, you either need to overcome the breakdown voltage of the medium between the electrodes, or break the electrical circuit.

The occurrence of an electrical discharge in the form of an electric arc in an uncontrolled environment, as in the case of an arc flash, can lead to injury, fire, equipment damage, and other accidents. These are, first of all, contact switching devices used in power supply and electric drive: high-voltage switches, circuit breakers, magnetic starters, contactors, sectional insulators on the contact network of electrified railways and urban electric transport, etc.. When disconnecting the loads of the above mentioned devices or breaking the electrical circuit, an arc arises between the opening contacts [1].

Experimental studies of such phenomena are complex and burdensome due to their temporal short duration, therefore, in many cases, only a mathematical model can give necessary and adequate information about their dynamics [1]. During the burning of an electric arc a lot of heat is released, a burning temperature of the electric arc reaches values of more than 3000 degrees Celsius. In high-voltage circuits, the arc length reaches a meter or more. As a result, there is a danger of harming human health and damage to equipment, as well as damage to environment.

Thermo-physical processes in the electric arc of high-current disconnecting devices are described by the physical model, the mathematical interpretation of which is the studied problem (1) - (3) [1], [2]. The formulation of the boundary value problem (1) - (3) is as follows. In the domain $\Omega=\{(x, t): \quad x \in(0, \infty) ; \quad t \in(0, \infty)\}$ the essentially loaded heat equation is investigated 


$$
\frac{\partial u}{\partial t}-\frac{\partial^{2} u}{\partial x^{2}}-\frac{1-2 \beta}{x} \cdot \frac{\partial u}{\partial x}-\left.\lambda \cdot \frac{\partial^{k} u}{\partial x^{k}}\right|_{x=\bar{x}(t)}=f(x, t)
$$

where $u=u(x, t)$ is an unknown function. $\beta, \lambda$ и $k$ are numerical parameters, and $0<\beta<1$, $\lambda \in C, k=0,1,\left.2 \ldots \lambda \cdot \frac{\partial^{k} u}{\partial x^{k}}\right|_{x=\bar{x}(t)}$ is a loaded summand, $f(x, t)$ is a known function defined in the domain $\Omega$. The load point moves according to a given law $x=\bar{x}(t)$ for $t \in(0, \infty)$, and the initial condition

a boundary condition

$$
u(x, 0)=g(x)
$$

$$
u(0, t)=h(t)
$$

are given. Functions $g(x)$ and $h(t)$ are given at $x \in(0, \infty)$ and $t \in(0, \infty)$ respectively.

The mathematical model in the form of the boundary value problem (1) - (3) is reduced to the Volterra integral equation of the second kind, as a result of which the solvability of the boundary value problem is equivalent to the solvability of the reduced integral equation.

\section{Reduced integral equation}

When finding solutions to the boundary value problem (1) - (3) for a essentially loaded differential parabolic equation, the necessity naturally arises to study the Volterra integral equation of the second kind of the following form

$$
\mu(t)-\lambda \cdot \int_{0}^{t} K_{k}(t, \tau) \cdot \mu(\tau) \cdot d \tau=F(t)
$$

where $\lambda \in C$ is the numerical parameter of the equation, $F(t)$ is the known function defined on the interval $(0, \infty), \mu(t)$ is the desired function. The kernel $K(t, \tau)$ of the integral equation (4) has the form

$$
K(t, \tau)=\left.\frac{\partial^{k} Q(x, t-\tau)}{\partial x^{k}}\right|_{x=\bar{x}(t)}
$$

where

$$
\begin{gathered}
Q(x, t-\tau)=\frac{x^{\beta}}{2(t-\tau)} \cdot \exp \left(-\frac{x^{2}}{4(t-\tau)}\right) \cdot P(x, t-\tau), \\
P(x, t-\tau)=\int_{0}^{\infty} \xi^{1-\beta} \exp \left(-\frac{\xi^{2}}{4(t-\tau)}\right) \cdot I_{\beta}\left(\frac{\xi \cdot x}{2(t-\tau)}\right) \cdot d \xi,
\end{gathered}
$$

and $x=\bar{x}(t)$ is the given function with $t \in(0, \infty), k$ is the order of the loaded term $(k=0,1,2 \ldots)$, $I_{\beta}(z)$ is the order of the loaded term.

\section{Various representations of the kernel-forming function $Q(x, t-\tau)$ and special cases of the kernel $K(t, \tau)$}

The function $Q(x, t-\tau)$ defines the kernel of the integral equation (4), and besides

$$
Q(x, t-\tau)=K_{0}(x, t) .
$$

As it is known, a type (a form) and the properties of the kernel play an important role in the question of solvability of the integral equation and dictate the methods of investigation of the 
integral equation. We calculate the function $Q(x, t-\tau)$ in general form and the kernel $K(t, \tau)$ in special cases and present their various types (interpretations) that will be used to determine the properties of the kernel and in the further study of the original boundary value problem (1) - (3).

1) Taking into account that [3]

$$
\int_{0}^{\infty} x^{\alpha-1} \exp \left(-p x^{r}\right) \cdot I_{v}(c x) d x=\frac{c^{v}}{2^{v} r \cdot p^{\frac{\alpha+v}{r}} \Gamma(v+1)} \sum_{n=0}^{\infty} \frac{1}{n !(v+1)_{n}} \Gamma\left(\frac{2 n+\alpha+v}{r}\right) \cdot\left(\frac{c}{2 p^{\frac{1}{r}}}\right)^{2 n},
$$

when $\operatorname{Re} p, \operatorname{Re}(\alpha+v)>0 ; r>1$, where

$$
(v+1)_{0}=1, \quad(v+1)_{n}=(v+1)(v+2) \cdot \ldots \cdot(v+n), n=1,2,3, \ldots
$$

are Pochhammer symbols, $\Gamma(z)$ is the gamma function, from (6) we receive the following representation for the function $Q(x, t-\tau)$

$$
Q(x, t-\tau)=\left(\frac{x}{2}\right)^{2 \beta} \frac{1}{\Gamma(\beta+1)(t-\tau)^{\beta}} \cdot \exp \left(-\frac{x^{2}}{4(t-\tau)}\right) \cdot \sum_{n=0}^{\infty} \frac{1}{(\beta+1)_{n}(t-\tau)^{n}} \cdot\left(\frac{x}{2}\right)^{2 n} .
$$

We calculate $K(t, \tau)(5)$ for $k=2$. To do this, we find $K(t, \tau)$ for $k=1$

$$
\begin{gathered}
\left.K(t, \tau)\right|_{\substack{k=1 \\
x=\bar{x}(t)}}=K_{1}(t, \tau)=\frac{\bar{x}^{2 \beta}}{2^{2 \beta-1} \Gamma(\beta+1)(t-\tau)^{\beta}} \cdot \exp \left(-\frac{\bar{x}^{2}}{4(t-\tau)}\right) \times \\
\times \sum_{n=0}^{\infty} \frac{\bar{x}^{2 n-1}}{2^{2 n}(\beta+1)_{n}(t-\tau)^{n}} \cdot\left(\beta+n-\frac{\bar{x}^{2}}{4(t-\tau)}\right) .
\end{gathered}
$$

Further, we obtain $K(t, \tau)$ for $k=2$

$$
\begin{aligned}
\left.K(t, \tau)\right|_{\substack{k=2 \\
x=\bar{x}(t)}}= & K_{2}(t, \tau)=\frac{\bar{x}^{2 \beta-1}}{2^{2 \beta-2} \Gamma(\beta+1)(t-\tau)^{\beta}} \cdot \exp \left(-\frac{\bar{x}^{2}}{4(t-\tau)}\right) \cdot \sum_{n=0}^{\infty} \frac{\bar{x}^{2 n-1}}{2^{2 n}(\beta+1)_{n}(t-\tau)^{n}} \times \\
& \times\left\{\frac{\bar{x}^{4}}{16(t-\tau)^{2}}-\left[2(\beta+n)+\frac{1}{2}\right] \cdot \frac{\bar{x}^{2}}{4(t-\tau)}+(\beta+n)\left(\beta+n-\frac{1}{2}\right)\right\} .
\end{aligned}
$$

2) For the function $Q(x, t-\tau)$, another a relation can be obtained using the integral representation of the modified Bessel function [4]

$$
I_{\beta}(z)=\frac{\left(\frac{z}{2}\right)^{\beta}}{\Gamma\left(\beta+\frac{1}{2}\right) \cdot \Gamma\left(\frac{1}{2}\right)} \cdot \int_{-1}^{1}\left(1-t^{2}\right)^{\beta-\frac{1}{2}} \cdot \exp ( \pm z t) d t, \quad \operatorname{Re}\left(\beta+\frac{1}{2}\right)>0
$$

the equality [4]

$$
\int_{0}^{\infty} x \cdot \exp \left(-\mu x^{2}-2 v x\right) \cdot d x=\frac{1}{2 \mu}-\frac{v}{2 \mu} \cdot \sqrt{\frac{\pi}{\mu}} \cdot \exp \left(\frac{v^{2}}{\mu}\right) \cdot\left[1-\Phi\left(\frac{v}{\sqrt{\mu}}\right)\right]
$$

when $|\arg v|<\frac{\pi}{2}, \operatorname{Re} \mu>0$; the relationship [5]

$$
\int_{0}^{a}\left(a^{\mu}-x^{\mu}\right)^{p-1} d x=\mu^{-1} \cdot a^{\mu(p-1)+1} \cdot B\left(p, \frac{1}{\mu}\right),
$$

where $a, \mu, \operatorname{Re} p>0$, and the ratio [3] 


$$
\begin{gathered}
\int_{0}^{a} x^{\alpha-1}\left(a^{2}-x^{2}\right)^{p-1} \exp \left(c^{2} x^{2}\right) \cdot\left\{\begin{array}{c}
\operatorname{erf}(c x) \\
\operatorname{erfc}(c x)
\end{array}\right\} d x= \\
= \pm \frac{a^{\alpha+2 p-1} c}{\sqrt{\pi}} B\left(\frac{\alpha+1}{2}, p\right) \cdot{ }_{2} F_{2}\left(1, \frac{\alpha+1}{2} ; \frac{3}{2}, p+\frac{\alpha+1}{2} ; a^{2} c^{2}\right)+ \\
+\left\{\begin{array}{l}
0 \\
1
\end{array}\right\} \frac{a^{\alpha+2 p-2}}{2} B\left(\frac{\alpha}{2}, p\right) \cdot{ }_{1} F_{1}\left(\frac{\alpha}{2} ; \frac{\alpha}{2}+p ; a^{2} c^{2}\right)
\end{gathered}
$$

for $a, \operatorname{Re} p>0 ; \operatorname{Re} \alpha>-\frac{1 \pm 1}{2}$, where

$$
\operatorname{erf}(z)=\frac{2}{\sqrt{\pi}} \int_{0}^{z} e^{-t^{2}} d t
$$

is the error function, moreover

$$
\operatorname{erfc}(z)=1-\operatorname{erf}(z)=\frac{2}{\sqrt{\pi}} \int_{z}^{\infty} e^{-t^{2}} d t
$$

$B(z)$ is a beta function;

$$
{ }_{p} F_{q}\left(a_{1}, \ldots, a_{p} ; b_{1}, \ldots b_{q} ; z\right)=\sum_{k=0}^{\infty} \frac{\left(a_{1}\right)_{k} \cdot \ldots \cdot\left(a_{p}\right)_{k}}{\left(b_{1}\right)_{k} \cdot \ldots \cdot\left(b_{p}\right)_{k}} \cdot \frac{z^{k}}{k !}
$$

is a generalized hyper-geometric function,

$$
{ }_{1} F_{1}(a ; b ; z)=\sum_{k=0}^{\infty} \frac{(a)_{k}}{(b)_{k}} \cdot \frac{z^{k}}{k !}
$$

is the degenerate hyper-geometric function.

After using the above equalities, the relation (7) takes the form

$$
P(x, t-\tau)=\frac{x^{\beta}(t-\tau)^{1-\beta}}{2^{2 \beta-1} \Gamma(\beta+1)} \pm \frac{x^{\beta+2}}{2^{2 \beta=1} \Gamma(\beta+2)(t-\tau)^{\beta}} \cdot{ }_{2} F_{2}\left(1, \frac{3}{2} ; \frac{3}{2}, \beta+2 ; \frac{x^{2}}{4(t-\tau)}\right) .
$$

Substituting (12) into (6), we obtain the following representation for the function $Q(x, t-\tau)$

$$
\begin{aligned}
& Q(x, t-\tau)=\exp \left(-\frac{x^{2}}{4(t-\tau)}\right) \cdot\left[\frac{x^{2 \beta}}{2^{2 \beta} \Gamma(\beta+1)} \cdot \frac{1}{(t-\tau)^{\beta}} \pm\right. \\
& \left. \pm \frac{x^{2 \beta+2}}{2^{2 \beta+2} \Gamma(\beta+2)} \cdot \frac{1}{(t-\tau)^{\beta+1}} \cdot{ }_{2} F_{2}\left(1, \frac{3}{2} ; \frac{3}{2}, \beta+2 ; \frac{x^{2}}{4(t-\tau)}\right)\right],
\end{aligned}
$$

3) Using (10) to find the function ${ }_{2} F_{2}\left(1, \frac{3}{2} ; \frac{3}{2}, \beta+2 ; \frac{x^{2}}{4(t-\tau)}\right)$

$$
{ }_{2} F_{2}\left(1, \frac{3}{2} ; \frac{3}{2}, \beta+2 ; \frac{x^{2}}{4(t-\tau)}\right)=\sum_{n=0}^{\infty} \frac{1}{(\beta+2)_{n}} \cdot\left(\frac{x^{2}}{4(t-\tau)}\right)^{n}
$$

and substituting the obtained relation in (13), we obtain the representation for the function $Q(x, t-\tau)$ in the form

$$
\begin{aligned}
& Q(x, t-\tau)=\exp \left(-\frac{x^{2}}{4(t-\tau)}\right) \cdot\left[\frac{x^{2 \beta}}{2^{2 \beta} \Gamma(\beta+1)} \cdot \frac{1}{(t-\tau)^{\beta}} \pm\right. \\
& \left. \pm \frac{x^{2 \beta+2}}{2^{2 \beta+2} \Gamma(\beta+2)} \cdot \frac{1}{(t-\tau)^{\beta+1}} \cdot \sum_{n=0}^{\infty} \frac{1}{(\beta+2)_{n}} \cdot\left(\frac{x^{2}}{4(t-\tau)}\right)^{n}\right]
\end{aligned}
$$


Now we calculate $K(t, \tau)$ when $k=2$ using a sequential differentiation of (14).

$$
\begin{gathered}
\left.K(t, \tau)\right|_{\substack{k=1 \\
x=\bar{x}(t)}}=K_{1}(t, \tau)=\frac{\bar{x}^{2 \beta}}{2^{2 \beta-1} \cdot(t-\tau)^{\beta}} \cdot \exp \left(-\frac{\bar{x}^{2}}{4(t-\tau)}\right) \times \\
\times\left\{\frac{1}{\Gamma(\beta+1)}\left[\frac{\beta}{\bar{x}}-\frac{\bar{x}}{4(t-\tau)}\right] \pm \frac{1}{\Gamma(\beta+2)} \sum_{n=0}^{\infty} \frac{\bar{x}^{2 n}}{2^{2 n+2}(\beta+2)_{n}(t-\tau)^{n+1}}\left[(\beta+n+1) \bar{x}-\frac{\bar{x}^{3}}{4(t-\tau)}\right]\right\} .
\end{gathered}
$$

For $Q(x, t-\tau)(13),(14)$ the kernel $K(t, \tau)$ when $k=2$ has the form

$$
\begin{gathered}
\left.K(t, \tau)\right|_{\substack{k=2 \\
x=\bar{x}(t)}}=K_{2}(t, \tau)=\frac{\bar{x}^{2 \beta}}{2^{2 \beta+1} \cdot(t-\tau)^{\beta+1}} \cdot \exp \left(-\frac{\bar{x}^{2}}{4(t-\tau)}\right) \times \\
\times\left\{\frac{1}{\Gamma(\beta+1)}\left[\frac{\bar{x}^{2}}{2(t-\tau)}-4 \beta-1+\frac{4 \beta(2 \beta-1)(t-\tau)}{\bar{x}^{2}}\right] \pm\right. \\
\left. \pm \frac{1}{\Gamma(\beta+2)} \sum_{n=0}^{\infty} \frac{\bar{x}^{2 n}}{2^{2 n}(\beta+2)_{n}(t-\tau)^{n}}\left[\frac{\bar{x}^{4}}{8(t-\tau)^{2}}-\frac{4(n+\beta)+5}{4(t-\tau)} \bar{x}^{2}+(\beta+n+1)(2(\beta+n)+1)\right]\right\} .
\end{gathered}
$$

4) If we use relation [3] to calculate function $P(x, t-\tau)$ (7),

$$
\int_{0}^{\infty} x^{\alpha-1} \exp \left(-p x^{2}\right) \cdot I_{v}(c x) \cdot d x=A_{v}^{\alpha}
$$

when $\operatorname{Re} p, \operatorname{Re}(\alpha+v)>0 ;|\arg c|<\pi$, where

$$
\begin{gathered}
A_{v}^{\alpha}=2^{-v-1} c^{v} p^{-\frac{\alpha+v}{2}} \frac{\Gamma\left(\frac{\alpha+v}{2}\right)}{\Gamma(v+1)} \cdot{ }_{1} F_{1}\left(\frac{\alpha+v}{2} ; v+1 ; \frac{c^{2}}{4 p}\right), \quad A_{v}^{2-v}=\frac{(2 p)^{v-1}}{c^{v} \Gamma(v)} \cdot \exp \left(\frac{c^{2}}{4 p}\right) \cdot \gamma\left(v, \frac{c^{2}}{4 p}\right), \\
\gamma(v, x)=\Gamma(v)-\Gamma(v, x)=\int_{0}^{x} t^{v-1} \cdot e^{-t} d t
\end{gathered}
$$

is the lower incomplete gamma function,

$$
\Gamma(v, x)=\int_{x}^{\infty} t^{\nu-1} \cdot e^{-t} d t
$$

is the upper incomplete gamma function, then from representation (7) we obtain

$$
P(x, t-\tau)=\frac{2(t-\tau)}{x^{\beta} \cdot \Gamma(\beta)} \cdot \exp \left(\frac{x^{2}}{4(t-\tau)}\right) \cdot \gamma\left(\beta, \frac{x^{2}}{4(t-\tau)}\right) .
$$

Substituting (17) into (6), we calculate $Q(x, t-\tau)$

$$
Q(x, t-\tau)=\frac{1}{\Gamma(\beta)} \cdot \gamma\left(\beta, \frac{x^{2}}{4(t-\tau)}\right) .
$$

After calculations, we have

$$
\left.K(t, \tau)\right|_{\substack{k=1 \\ x=\bar{x}(t)}}=K_{1}(t, \tau)=\frac{1}{\Gamma(\beta) \cdot 2^{2 \beta-1}} \cdot \frac{\bar{x}^{2 \beta-1}}{(t-\tau)^{\beta}} \cdot \exp \left(-\frac{\bar{x}^{2}}{4(t-\tau)}\right) .
$$

Next, we calculate the kernel $K(t, \tau)$ when $k=2$ for the integral equation (4) by making similar calculations, 


$$
\left.K(t, \tau)\right|_{\substack{k=2 \\ x=\bar{x}(t)}}=\frac{\bar{x}^{2 \beta-2}}{2^{2 \beta-1} \Gamma(\beta)(t-\tau)^{\beta}} \cdot \exp \left(-\frac{\bar{x}^{2}}{4(t-\tau)}\right) \cdot\left[2 \beta-1-\frac{\bar{x}^{2}}{2(t-\tau)}\right] .
$$

5) Using (16), (18), the function $Q(x, t-\tau)$ can also be represented as

$$
Q(x, t-\tau)=1-\frac{1}{\Gamma(\beta)} \cdot \Gamma\left(\beta, \frac{x^{2}}{4(t-\tau)}\right) .
$$

Obviously, the kernel $K(t, \tau)$ when $k=2$ has the form (19).

6) Taking into consideration the ratio [3]

$$
\gamma(v, x)=\frac{z^{v}}{v} \cdot{ }_{1} F_{1}(v ; v+1 ;-z)
$$

and relation (11), we transform the representation (18) to the form

$$
Q(x, t-\tau)=\frac{1}{\Gamma(\beta)} \cdot \frac{x^{2}}{2^{2 \beta}(t-\tau)^{\beta}} \cdot \sum_{n=0}^{\infty} \frac{(-1)}{(\beta+n) \cdot n !} \cdot\left(\frac{x^{2}}{4(t-\tau)}\right)^{n} .
$$

Next we compute the kernel $K(t, \tau)$ under the condition $k=2$

$$
\left.K(t, \tau)\right|_{\substack{k=2 \\ x=\bar{x}(t)}}=\frac{1}{2^{2 \beta-1} \Gamma(\beta)(t-\tau)^{\beta}} \cdot \sum_{n=0}^{\infty} \frac{(-1)^{n} \cdot(2 n+1)(n+1)}{(\beta+n) \cdot n ! \cdot(t-\tau)^{n} \cdot 2^{2 n}} \cdot \bar{x}^{2 n} .
$$

7) Taking into account that [3]

$$
\gamma(v+1, z)=v \cdot \gamma(v, z)-z^{v} e^{-z}
$$

we obtain from (18) for the function $Q(x, t-\tau)$ a representation in the form [6]

$$
Q(x, t-\tau)=\frac{1}{\Gamma(\beta)} \cdot\left[(\beta-1) \cdot \gamma\left(\beta-1, \frac{x^{2}}{4(t-\tau)}\right)-\left(\frac{x}{2}\right)^{2 \beta-2}(t-\tau)^{1-\beta} \exp \left(-\frac{x^{2}}{4(t-\tau)}\right)\right] .
$$

Since (23) is obtained from (18), the kernel $K(t, \tau)$ when $k=2$ has the form (19), as you can see by direct differentiation.

\section{Properties of the kernel-forming function $Q(x, t-\tau)$}

We list some properties of the function $Q(x, t-\tau)$, necessary for our research.

1) The function $Q(x, t-\tau), 0<\tau<t<\infty$, is continuous function.

2) The function $Q(x, t-\tau) \geq 0,0<\tau<t<\infty$ [1].

3) For function $Q(x, t-\tau)$, an estimate

$$
Q(x, t-\tau) \leq \frac{1}{\Gamma(\beta+1)} \cdot\left(\frac{x^{2}}{4(t-\tau)}\right)^{\beta} .
$$

takes place. The estimate (24) can be obtained in two ways: from the representation (18) and from the representation (8) for the function $Q(x, t-\tau)$.

4) For the function $Q(x, t-\tau)$, there is an inequality

$$
\begin{gathered}
Q(x, t-\tau)<\frac{1}{\Gamma(\beta+1)(t-\tau)^{\beta}} \cdot\left(\frac{x}{2}\right)^{2 \beta} \exp \left(-\frac{x^{2}}{4(t-\tau)}\right) \pm \\
\pm \frac{1}{\Gamma(\beta+2)(t-\tau)^{\beta+1}} \cdot\left(\frac{x}{2}\right)^{2 \beta+2} \exp \left(-\frac{x^{2}}{4(t-\tau)}\right) \pm \frac{1}{\Gamma(\beta+2)(t-\tau)^{\beta+2}} \cdot\left(\frac{x}{2}\right)^{2 \beta+4} .
\end{gathered}
$$

This inequality (25) is received from the representation (14) of the function $Q(x, t-\tau)$.

5) The following integral representation 


$$
\int_{0}^{t} Q(x, t-\tau) d \tau=\frac{1}{\Gamma(\beta)} \cdot\left[t \cdot \gamma\left(\beta, \frac{x^{2}}{4 t}\right)+\frac{x^{2}}{4} \cdot \Gamma\left(\beta-1, \frac{x^{2}}{4 t}\right)\right] .
$$

holds for the function $Q(x, t-\tau)$. The relation (26) is derived from the representation (18) for the function $Q(x, t-\tau)$.

6) The function $Q(x, t-\tau)$ satisfies the following relation

$$
\lim _{t \rightarrow 0} \int_{0}^{t} Q(x, t-\tau) d \tau=0 .
$$

In fact, passing to the limit as $t \rightarrow 0$ in (26), we obtain the desired relation (27).

The required ratio (27) for the function $Q(x, t-\tau)$ can be obtained in a simpler way, for this purpose the limit $\lim _{t \rightarrow 0} \int_{0}^{t} Q(x, t-\tau) d \tau$ is calculated using the estimate (24) [7].

Here we have presented those interpretations (8), (13), (14), (18), (20), (21), (23), properties (24) - (27) functions $Q(x, t-\tau)$ in general case and types (9), (15), (19), (22) of the kernel $K(t, \tau)$ in particular cases, which are most convenient in studies of the integral equation (1), and, consequently, in further studies of the boundary value problem (1) - (3) for various specified values of parameters and for a specified law of motion $x=\bar{x}(t)$ of the load point.

\section{Conclusion}

1. For the boundary-value problem (1) - (3) where $\bar{x}(t)=t^{\omega}, \omega \in R$, in [1] solvability issues were determined and solutions were obtained in cases, when $k=0$ and $k=1$ for any values of $\omega$, and when $k=2$ for $\omega<\frac{1}{2}$.

It is known that the boundary-value problem is completely investigated if solvability issues are determined and solutions are obtained for any parameter values from the domain of their determination.

2. As we have shown in [1], the solvability of the boundary value problem (1) - (3) is equivalent to the solvability of integral equation (4). It was also shown in [1] that in case $k=2$ for $\omega \geq \frac{1}{2}$, the integral equation (4) is a special Volterra integral equation, which requires a specific research. In the study of the special Volterra integral equation of the second kind, the following tasks are set: to investigate its solvability, and to investigate spectral questions for this special Volterra integral equation of the second kind [8], [9]. The shape and type of the kernel determine how to solve these problems.

3. In the general case, when solving mathematical models of applied problems that describe physical, mechanical, and other processes or phenomena, preference is given to obtaining an exact analytical solution in comparison with graphic, approximate, experimental, and other solutions.

Thus, before investigating the solvability issues and obtaining analytical solutions for the parameters $k=2$ in the case $\omega \geq \frac{1}{2}$, as well as in the future for $k=3,4 \ldots$, when $\omega \in R$, due to the bulkiness of the kernel (2) of the integral equation (1), it is necessary to carry out mathematical calculations that simplify the further study of the boundary value problem (1) - (3).

These mathematical calculations, representing various representations and properties of the kernel-forming function $Q(x, t-\tau)$, are carried out in general case, and the kernel $K(x, t-\tau)$ is calculated in the special cases for further research to obtain an analytical solution of the boundary value problem (1) - (3) for the remaining parameter values. 
M.I. Ramazanov [2] completely investigated boundary value problems for this essentially loaded heat equation in the special case $\beta=\frac{1}{2}$. In contrast to these previous studies on the theory of loaded differential equations, in this paper boundary value problem for the essentially loaded heat equation (1) is considered in an unlimited domain in the general case with $0<\beta<1$.

\section{REFERENCES}

1 Yesbaev A.N., Yessenbayeva G.A., Ramazanov M.I. Investigation of the model for the essentially loaded heat equation. Eurasian Physical Technical Journal. 2019, Vol. 16, No. 1(31), pp. 113 - 120.

2 Dzhenaliev M.T., Ramazanov M.I. Loaded equations as perturbations of differential equations. Almaty, Publishing house «Gylym», 2010, 334 p.

3 Prudnikov A.P., Brychkov Yu.A., Marychev O.I. Integrals and series. Vol. 2. Special functions, Moscow, Fizmatlit, 2003, 664 p.

4 Gradshtein I.S., Ryzhik I.M. Tables of integrals, sums, series and products. Moscow, 1963, 1108 p. [in Russian]

5 Prudnikov A.P., Brychkov Yu.A., Marychev O.I. Integrals and series. Vol. 1. Elementary functions, Moscow, Fizmatlit, 2002, 632 p.

6 Yesbayev A.N., Yessenbayeva G.A. On the integral equation of the boundary value problem for the essentially loaded differential heat operator. Bulletin of Karaganda University. Mathematics Series. 2016, No. 3(83), pp. $62-69$.

7 Yesbayev A.N., Yessenbayeva G.A., Ramazanov M.I. The research of one boundary value problem for the loaded differential operator of heat conduction. Bulletin of Karaganda University. Mathematics Series. 2013, No. 3(71), pp. $35-42$.

8 Polyanin A.D., Manzhirov A.V. Spravochnik po integral'nym uravneniyam: Metody resheniya. Moscow, Publishing House "Factorial Press", 2000, 384 p. [in Russian]

9 Polyanin A.D., Manzhirov A.V. Spravochnik po integral'nym uravneniyam. Moscow, Fizmatlit, 2003, 608 p. [in Russian]

Article accepted for publication 09.12.2019 